\title{
Classifying Crystals of Rounded Tetrahedra and Determining Their Order Parameters Using Dimensionality Reduction
}

\author{
Robin van Damme,* Gabriele M. Coli, René van Roij, and Marjolein Dijkstra*
}

Cite This: ACS Nano 2020, 14, 15144-15153

Read Online

ACCESS

Llll Metrics \& More

Article Recommendations

Supporting Information

ABSTRACT: Using simulations we study the phase behavior of a family of hard spherotetrahedra, a shape that interpolates between tetrahedra and spheres. We identify 13 close-packed structures, some with packings that are significantly denser than previously reported. Twelve of these are crystals with unit cells of $N=2$ or $N=4$ particles, but in the shape regime of slightly rounded tetrahedra we find that the densest structure is a quasicrystal approximant with a unit cell of $N=82$ particles. All 13 structures are also stable below close packing, together with an additional 14th plastic crystal phase at the sphere side of the

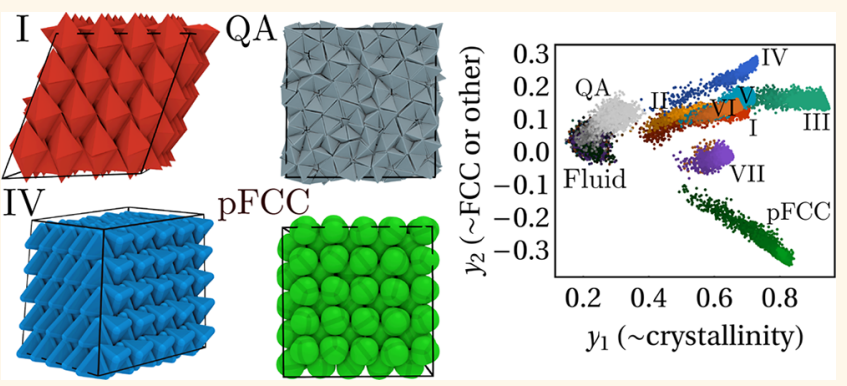
phase diagram, and upon sufficient dilution to packing fractions below 50-60\% all structures melt. Interestingly, however, upon compressing the fluid phase, self-assembly takes place spontaneously only at the tetrahedron and the sphere side of the family but not in an intermediate regime of tetrahedra with rounded edges. We describe the local environment of each particle by a set of $l$-fold bond orientational order parameters $\bar{q}_{l}$ which we use in an extensive principal component analysis. We find that the total packing fraction as well as several particular linear combinations of $\bar{q}_{l}$ rather than individual $\bar{q}_{l}$ 's are optimally distinctive, specifically the differences $\bar{q}_{4}-\bar{q}_{6}$ for separating tetragonal from hexagonal structures and $\bar{q}_{4}-\bar{q}_{8}$ for distinguishing tetragonal structures. We argue that these characteristic combinations are also useful as reliable order parameters in nucleation studies, enhanced sampling techniques, or inversedesign methods involving odd-shaped particles in general.

KEYWORDS: tetrahedra, self-assembly, quasicrystal, dimensionality reduction, machine learning, nanoparticles, computer simulation.

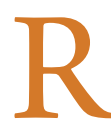

ecent advances in colloid synthesis allow for a plethora of particle shapes from the nanometer to the micrometer scale. ${ }^{1-7}$ These particles have a Brownian character when suspended in a liquid such as water or toluene, which causes self-assembly into a huge variety of ordered structures at sufficiently high concentrations. These selfassembled structures can form materials with useful optical, ${ }^{8}$ photonic, ${ }^{9}$ electronic, ${ }^{10}$ or catalytic ${ }^{11}$ properties. Simulations and experiments of monodisperse systems of sterically stabilized particles, for instance, exhibit a wealth of densestpacked structures that are far more complex than the wellknown face-centered-cubic (FCC) crystal phase for spheres and the simple cubic crystal for cubes. ${ }^{12-17}$ Interestingly, the study of close-packed structures of rigid hard bodies has a long history with relevance far beyond nanoparticle self-assembly; it goes back to Kepler's stacking of cannonballs in $1611^{18}$ and the packing of sand piles in more recent times. ${ }^{19}$ During the past years, various simulation methods have been employed to predict the self-assembled structures and densest crystals for hard-particle systems such as tetrahedra, ${ }^{20-24}$ Platonic solids, ${ }^{12,20,22,24}$ rounded cubes, ${ }^{25-29}$ cuboctahedra, ${ }^{13,15,16,30}$ truncated tetrahedra, ${ }^{31}$ and even families of shapes without conventional names. ${ }^{22,24,32-34}$ Despite this tremendous progress in predicting densest packings and ordered structures of odd-shaped particles, characterization and classification remain difficult. In fact, the relevant order parameters to distinguish the various solid phases are often not even known in advance. For instance, in simulations of truncated cubes no fewer than 14 crystal phases were identified on the basis of lattice vectors and lattice angles of the unit cell, but crystal distortions and degeneracies obscured a clear-cut classification. ${ }^{15,16}$

Received: June 26, 2020

Accepted: October 16, 2020

Published: October 26, 2020 
Inspired by recent experiments on faceted tetrahedral nanoparticles ${ }^{35}$ coated with either ligands $s^{36,37}$ or possibly a shell of silica, ${ }^{38,39}$ we study in this paper the phase behavior of a system consisting of hard spherotetrahedra, a shape that interpolates between tetrahedra and spheres. We focus first on the (possibly quasi-)crystalline densest packings, before turning to the crystal structures at intermediate densities, followed by a closer look at how dimensionality reduction can provide reliable order parameters for distinguishing these structures.

\section{RESULTS AND DISCUSSION}

Model System. We consider a one-component system of hard spherotetrahedra. This is the shape one obtains by considering the volume within a distance $R$ from a tetrahedron of edge length $L$. Numerically, we define it by the Minkowski sum of a tetrahedron of edge length $L$ and a sphere of radius $R$, as illustrated in Figure 1. It is fully characterized by a shape

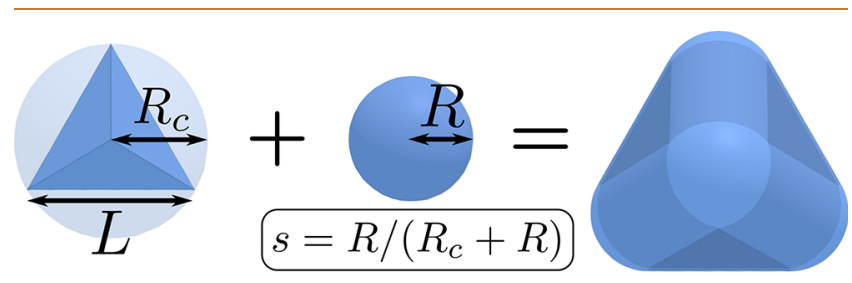

Figure 1. A spherotetrahedron is the Minkowski sum of a tetrahedron (edge length $L$ and circumscribed radius $R_{c}=\sqrt{6} L / 4$ ) and a sphere (radius $R$ ). The shape is fully described by the dimensionless shape parameter $s$.

parameter $s=R /\left(R_{c}+R\right) \in[0,1]$, with $R_{c}=\sqrt{6} L / 4$ the radius of the circumscribed sphere around the tetrahedron. The limiting cases describe a tetrahedron $(s=0)$ and a sphere $(s=1)$, so $s$ can also be interpreted as the roundness.

Densest Packings. The densest packing of hard spheres is the FCC crystal with a well-established maximum packing fraction of $\phi_{\max }=\pi /(3 \sqrt{2}) \approx 0.74 .^{40}$ The best-packed structure of tetrahedra was the subject of a flurry of publications some years back, ${ }^{12,20,21,23,41-46}$ when numerical simulations revealed that a dodecagonal quasicrystal forms spontaneously from the fluid via a first-order phase transition and that a quasicrystal approximant can be compressed to a packing fraction as high as $\phi=0.8503 .{ }^{21,23}$ This raised the conjecture that the densest packing of tetrahedra might be aperiodic, although later work showed that the periodic dimer crystal $^{43,45}$ achieves a packing fraction of $\phi_{\max }=4000 / 4671=$ 0.856348 , which is the established densest packing of tetrahedra as of now.

For slightly rounded spherotetrahedra, Jin et al. ${ }^{47}$ surprisingly found that a quasicrystal approximant packs denser than the dimer crystal structure. This fascinating result inspired us to reinvestigate in more detail the densest packings of spherotetrahedra in the full shape range of $s \in[0,1]$.

To obtain the densest packings of spherotetrahedra as a function of the shape parameter $s$, we use the floppy-box Monte Carlo (FBMC) algorithm described in ref 24. This algorithm finds the densest packings of hard particles by numerically compressing unit cells of variable shapes with periodic boundary conditions and a small number of particles to increasingly high pressures. For a given particle shape we perform at least 20 independent compression runs, where we use different compression routes to improve the sampling. Figure 2 shows typical snapshots of close-packed structures that we identified in this way, including their primitive unit cell with the relative orientations of the $N=2$ or 4 particles. In Figure 3(a) we present the resulting maximum packing fraction $\phi_{\max }$ as a function of the shape parameter $s$ along with those of ref 47 for comparison. We reproduce the well-known limiting close-packed densities for $s=0$ and $s=1$, but obtain substantially higher maximum packing fractions for intermediate shapes than previously reported. All structures we find satisfy Ulam's conjecture, which states that all nonsphere convex shapes pack denser than spheres (i.e., $\left.\phi_{\max }>\pi /(3 \sqrt{2}) \approx 0.74\right)$.

Crystal Classification. The vertical solid black lines in Figure 3 divide the shape parameter space into 12 regions labeled by Roman numerals corresponding to 12 structurally distinct dense packings. Some of these structures, e.g., III", III', and III, are extremely similar and only distinct in the closestpacking limit. We therefore label these together as below dense packing only a single structure exists, which we then simply refer to as III.

Previous work has distinguished crystal structures based on discontinuities in the derivatives of the shape-dependent maximum packing fraction $\phi_{\max }(s)^{31,48}$ or in the lengths $L_{i}$ of and the angles $\theta_{i}$ between the three vectors that span the unit cell, $^{15}$ which are shown in Figure $3(\mathrm{a}),(\mathrm{b})$, and (c), respectively. Both these methods have important drawbacks. Discontinuities in the derivatives of the maximum packing fraction can indeed be the result of jumps from one crystal structure to another, but can also be the result of a change in continuous deformation of the lattice, e.g., from a shear along one axis to shear along another. The lattice parameters do not uniquely describe a crystal structure and can be degenerate (regions I, II, VII", and $\mathrm{VII}^{\prime}$ in Figure 3(b) and (c)). Moreover, the analysis of lattice parameters of the unit cell is inherently not extendable to aperiodic structures such as quasicrystals, fluids, mesophases, glasses, etc. For these reasons we instead define the regions I-VII through discontinuities in a set of averaged Steinhardt bond orientational order parameters $\bar{q}_{v}{ }^{49,50}$ with modifications proposed by ref 51 to obtain a fully parameter-free description. In this way, the set of all $\bar{q}_{l}$ forms a unique "fingerprint" for each crystal structure. In practice, one only needs a few $\bar{q}_{l}$, s in order to distinguish all dense packings from each other. We show the subset $\left\{\bar{q}_{2}, \bar{q}_{4}, \ldots\right.$, $\left.\bar{q}_{12}\right\}$ in Figure 3(d). First-order discontinuities occur in all $\bar{q}_{l}$ 's simultaneously whenever there is a jump from one structure to another and define the vertical solid black line boundaries, while second-order discontinuities correspond to changes in the lattice type as the crystals deform, e.g., along different planes as the shape changes. Zoom-ins for various regions of interest in Figure 3 are provided in the SI.

Crystal Structures. The crystal structures I-VII can be loosely grouped as deformed dimer (I, II), deformed $\beta$-tin $\left(\mathrm{IV}^{\prime}, \mathrm{IV}\right)$, and deformed FCC (VII", VII', VII), with intermediate structures (III", III', III, V, VI). For $s \approx 0$, close to tetrahedra, we find two very similar dimer crystals I and II: both are triclinic lattices with $N=4$ particles in a unit cell in which the tetrahedra form dimers with almost perfect facet-to-facet alignment. The difference between the two (shown in Figure S11) is a subtle shift in the relative position and orientation of the two dimers with respect to one another. The particles in the crystal structures III"-VII do not form 

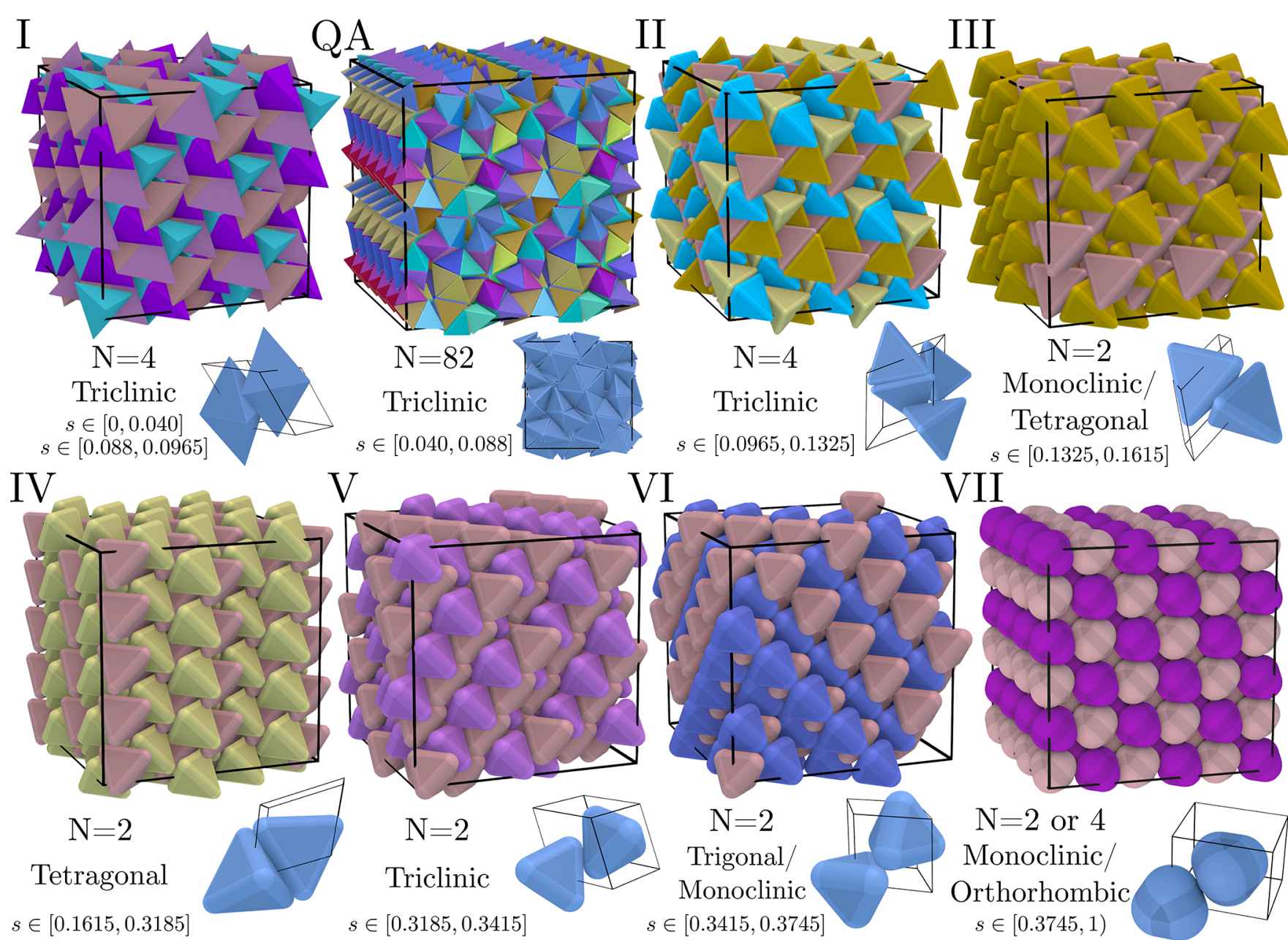

VII

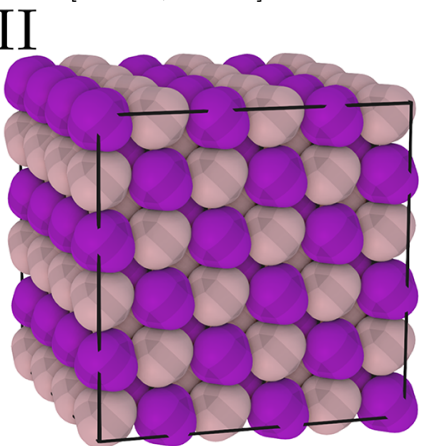

$s \in[0.1615,0.3185]$

$s \in[0.3185,0.3415]$

$s \in[0.3415,0.3745]$

Figure 2. Cubic supercells (with distinct colors indicating differently oriented particles in the unit cell) and primitive unit cells of eight of the densest crystal structures, shown with their lattice type and the shape-parameter s-regime in which they are the densest packing. Roman numerals denote crystal structures obtained from the floppy-box Monte Carlo simulations, and "QA" indicates the quasicrystal approximant.

dimers, but instead align with respect to one another rotated by $60^{\circ}$ around a common facet normal. Each of these crystal structures has its own noteworthy properties. In crystal structures III", III', and III particles have an unusually high 20 Voronoi neighbors (SI, Figure S4). Additionally, at $s=$ 0.1465 its lattice type switches from monoclinic to tetragonal. Crystal structures IV' and IV bear a close resemblance to the $\beta$-tin lattice found for truncated tetrahedra in ref 31 : it is tetragonal and at a rounding ratio of $s=0.178$ it packs as efficiently as $\phi=0.90212$, which is the densest of all spherotetrahedra. While crystal phase IV is stable over a large range of shapes, its packing efficiency decreases with increasing roundness $s$, until it is superseded by crystal structure $\mathrm{V}$, a triclinic transition structure that is only stable over a small range of shapes $(s \in[0.3185,0.3415])$. This is followed by crystal structure VI, which is trigonal for $s<0.36$ and monoclinic for $s \geq 0.36$. The trigonal region $s \in[0.3415,0.36]$ is the only region for all spherotetrahedra for which a change in the particle shape does not change the lattice parameters and only barely changes the structure of the closest-packed crystal. Finally, crystals VII", VII', and VII are deformations of an FCC lattice. To achieve the densest packing, the lattice becomes orthorhombic rather than cubic for $s \in[0.4136,1]$ and requires two particles in the aforementioned opposing orientations. For $s \in[0.3745,0.4135]$ there are additional changes: here the even denser packings VII" and VII' can be achieved by four-particle unit cells if the two additional particles are slightly rotated (SI Figure S3) and the lattice symmetry is reduced from orthorhombic to monoclinic.

As previous studies report that more centrally symmetric particles tend to pack into simpler lattices, ${ }^{12,31}$ we might expect the densest packings of spherotetrahedra to become simpler and with fewer particles in the unit cell with increasing shape parameter $s$. Interestingly, this trend does not hold here. While the number of particles per unit cell initially decreases from $N$ $=4$ to $N=2$ when the shape parameter increases up to $s=$ 0.3745 , this trend is broken in the region $s \in[0.3745,0.4135]$, where the crystal structures VII" and VII' require $N=4$ particles to obtain their densest packing. The densest packing can also become less symmetric as the shape becomes more spherical: while the crystal structures up to IV indeed become more symmetric, crystal $\mathrm{V}$ is again triclinic. In general, while we could expect that making particles more spherical will yield more symmetric lattices at intermediate densities due to entropy considerations, it appears that these symmetries may need to be broken in order to achieve the densest packing.

Maximum Packing Fraction of the Quasicrystal Approximant. Using the floppy-box Monte Carlo method, we found a variety of crystal structures of hard spherotetrahedra with a significantly higher maximum packing fraction than 


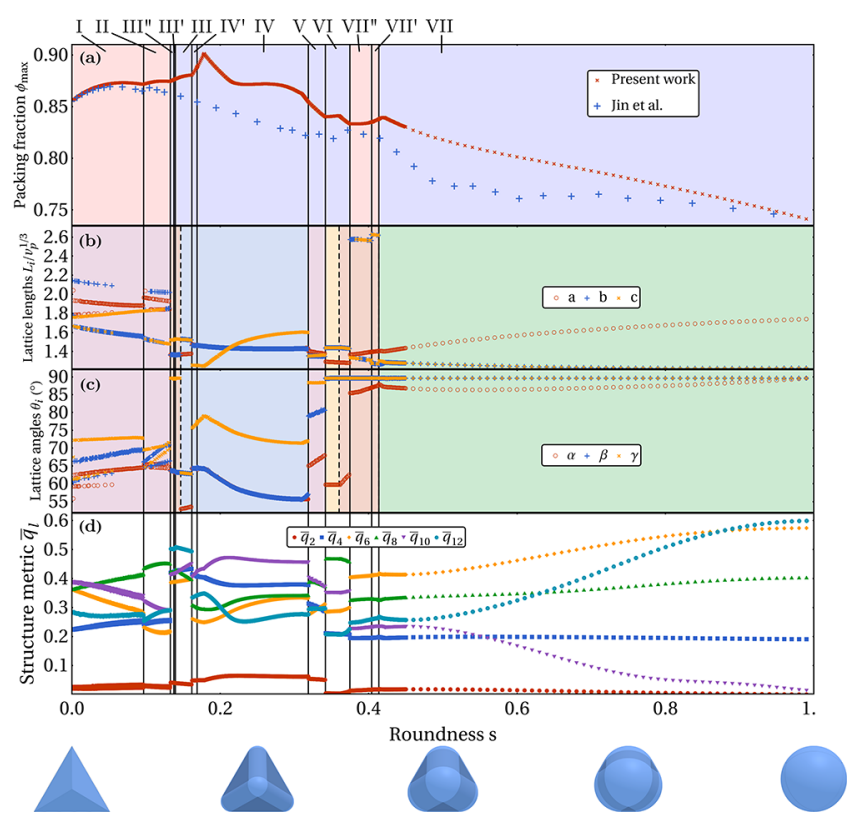

Figure 3. Floppy-box Monte Carlo simulation results of the shapeparameter dependence of (a) the maximum packing fractions $\phi_{\max }$ (b) the lengths $L_{i}$ of the lattice vectors, (c) the lattice cell angles $\theta_{i}$, and (d) the (modified) bond order parameters $\bar{q}_{l}$ for $l=2,4, \ldots, 12$ (see text), all for the densest structure we have found. Roman numerals denote the different crystal structures, and vertical solid black lines indicate the transitions between these structures. In (a) the red and blue backgrounds indicate four- and two-particle unit cells, respectively, and in (b) and (c) different background colors denote the different lattice types also noted in Figure 2. The dashed lines in (b) and (c) indicate transitions in lattice symmetry.

previously found in the literature. We now investigate whether these densest-packed crystal structures have a better packing than the quasicrystal approximant found in ref 47 . To this end, we first refine our search in the region $s \in[0.02,0.12]$ to 200 compression runs per shape parameter value $s$. In addition, we increase the total number of $\mathrm{MC}$ cycles to $M=10^{8}$ in our floppy-box Monte Carlo method and investigate unit cells with $4 \leq N \leq 20$ particles. Despite these refinements, we find that the $N=4$ dimer crystals I and II remain the densest crystal structures for $s \in[0.02,0.12]$. Subsequently, we determine the maximum packing fraction of the quasicrystal approximant reported in ref 47 by further compressing the unit cell of the quasicrystal approximant of $N=82$ particles. In Figure 4, we plot the maximum packing fraction for the quasicrystal approximant obtained in this work and the earlier results from ref 47 (large orange and blue dots, respectively) along with the maximum packing fractions of the crystal structures with a unit cell of $N \leq 20$ particles (small red dots). We make the following observations. In comparison with previous results, ${ }^{47}$ we find higher packing fractions for the quasicrystal approximant for $s \in[0.065,0.115]$, but slightly lower densities for $s \in[0.02,0.06]$. More interestingly, we find that the maximum density of the approximant surpasses that of the smaller unit cells for $s \in[0.04,0.088]$, thereby demonstrating that the quasicrystal approximant is the best-packed structure in this regime. This is highly surprising, since all the densest packings reported in the literature have unit cells with $N \leq$ $4,{ }^{12,15,20,22,26,32,52,53}$ making the $N=82$ approximant the largest unit cell for a densest packing of a single-component hardparticle system to date. This finding raises the fascinating

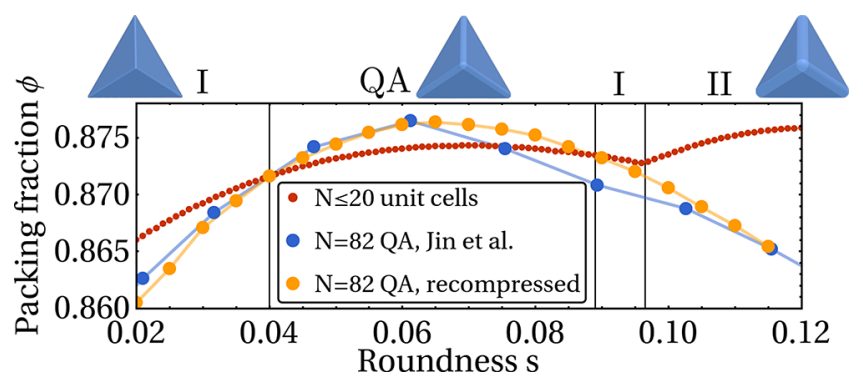

Figure 4. Maximum packing fraction of hard spherotetrahedra as a function of the roundness $s$ for the densest-packed crystal structures obtained from floppy-box Monte Carlo simulations using unit cells with $N \leq 20$ particles (red dots) and for the quasicrystal approximant reported by Jin et al., ${ }^{47}$ where previous and our results are denoted by blue and orange dots, respectively. In the region $s \in[0.040,0.088]$, the quasicrystal approximant is denser than the dimer crystal.

question whether quasicrystals with truly aperiodic order are the densest packings of spherotetrahedra in this shapeparameter regime. One may investigate this by determining the maximum density for quasicrystalline approximants with increasingly larger unit cells. Unfortunately, this is beyond the scope of this work, as it is presently unclear how to systematically construct unit cells of even larger quasicrystal approximants. Note, however, that such an approach was performed in the case of hard tetrahedra, ${ }^{23}$ where it was found that a quasicrystal approximant of $N=1142$ particles does not necessarily correspond to a higher density than an approximant with $N=82$. Systematic work along these lines is needed to resolve this issue.

Phase Behavior at Intermediate Densities. To investigate the full phase behavior of hard spherotetrahedra as a function of density, we perform Monte Carlo simulations in the canonical ensemble using the disordered fluid phase as the initial configuration. We use a relatively large number of Monte Carlo cycles, $M=10^{8}$, to study the self-assembly from the fluid phase, as previous simulations showed that the formation of a quasicrystal of hard tetrahedra is a slow process. $^{21,23,54}$ Additionally, we perform Monte Carlo simulations in a constant-pressure ensemble to measure the equations of state, i.e., the pressure as a function of density, of the fluid and solid phases. From the equations of state, we determine the phase boundaries for the different crystal structures and the fluid-solid coexistence regions. Figure 5 shows the resulting phase diagram of hard spherotetrahedra in the shape parameter $s$ versus packing fraction $\phi$ representation. For shapes close to tetrahedra $(s \in[0,0.16])$, a quasicrystal self-assembles from the fluid phase. Since the densest packing for $s \in[0.040,0.088]$ is the quasicrystal approximant, it is likely that quasicrystalline order is stable all the way from the fluid up to the maximum packing. However, the approximant and the quasicrystal are not strictly identical, and there may be a transition from one to the other at a certain density. Finding this transition is beyond the scope of this investigation as it is neither straightforward to construct quasi-crystal approximants with increasingly large unit cells nor to calculate the free energy of quasicrystals, as it requires an unknown configurational entropy contribution.

For $s \in[0.32,0.36]$ and $\phi \approx 0.62$, we find that the structures V and VI do not melt directly into a fluid, but instead follow the sequence $\mathrm{V} \rightarrow \mathrm{VI} \rightarrow \mathrm{VII} \rightarrow$ fluid; that is, the 


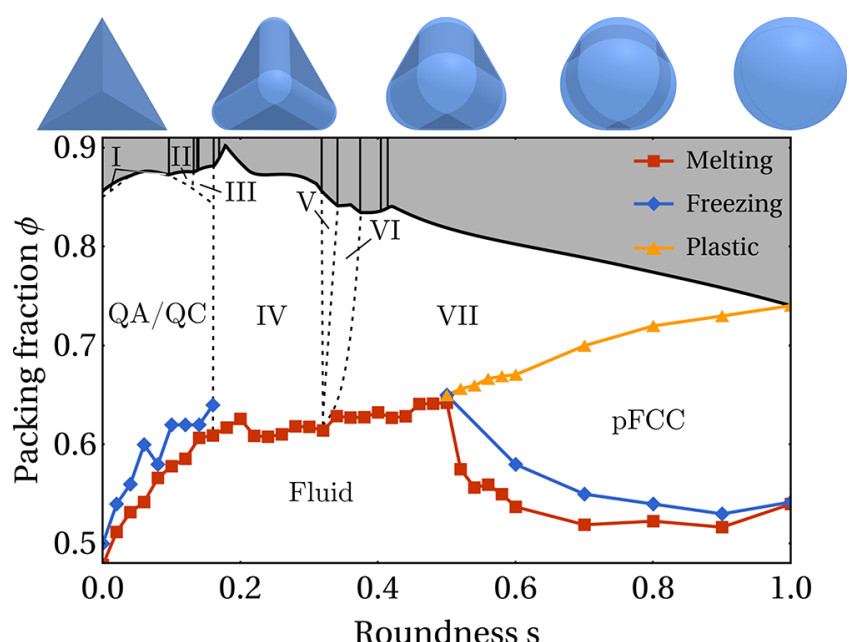

Figure 5. Phase diagram of hard spherotetrahedra in the shape parameter $s$ and packing fraction $\phi$ representation, with the forbidden region beyond close packing in gray. Solid lines in the forbidden region denote transitions between densest packings. Symbols (connected by a solid line to guide the eye) are determined from discontinuities in the equations of state, and dotted lines indicate estimates for the phase boundaries between the solid phases. For $0.16<s<0.5$, only the melting line is shown, as crystallization from the fluid phase was not observed.

less symmetric crystal structures first transform via solid-solid transitions into more symmetric structures before fully melting. This motivates the curved dotted lines in Figure 5. Despite the aforementioned high number of Monte Carlo cycles, we do not observe any crystallization whatsoever in the region $s \in(0.16$, $0.5)$. Furthermore, the melting density of the crystals in this region is relatively high, about $\phi \approx 0.6$. This absence of selfassembly was also found for similarly shaped truncated tetrahedra, ${ }^{31,33}$ which was attributed to the competition of multiple local structural motifs. ${ }^{34}$

We did observe self-assembly in the region $s \in[0.5,1.0]$, where a plastic crystal ( $\mathrm{pFCC}$ ) forms spontaneously from the disordered fluid state. In addition, the deformed FCC crystal VII melts into this plastic crystal upon lowering the pressure. The range of densities for which this plastic crystal is found increases with $s$. This is to be expected as unhindered particle rotations, and hence plastic crystals, become increasingly favorable for particles that approach the spherical shape.

Finding Order Parameters Using Principal Component Analysis. In the previous sections, we have shown that the phase diagram of hard spherotetrahedra exhibits a rich variety of crystal structures as shown in Figures 2, 3, and 5. We have identified, distinguished, and characterized these crystals by analyzing the equations of state, the lattice parameters of the unit cells, and a large set of bond order parameters $\bar{q}_{l}$ with $2 \leq l \leq 12$. However, in most studies either a single or a small set of suitably chosen order parameters, e.g., the density in the case of a gas-liquid transition or $\bar{q}_{6}$ for a fluid-solid transition of spheres, ${ }^{50,55}$ is employed in order to locate a phase transition or to bias the sampling to overcome free-energy barriers and to study nucleation. Additionally, order parameters may also be used to inverse-design a target structure. ${ }^{56}$ In general, finding the optimal set of order parameters to distinguish the different phases from one another may be very challenging.
Here, we develop a method to find the relevant set of order parameters that enables us to describe the various phases. To this end, we describe the local structural symmetries of all the particles in the different thermodynamic phases in terms of a large set of bond order parameters $\bar{q}_{l}$. Subsequently, we investigate which of the bond order parameters are redundant, and how many order parameters are required to distinguish the different phases at hand. For the complex crystal structures of spherotetrahedra, we observe from Figure 3(d) that many $\bar{q}_{l}$ 's are nonzero for the close-packed crystals. It is hence not $a$ priori clear which bond order parameters are required or are redundant. Below, we investigate to what extent all the information on the $\bar{q}_{l}$, s is needed to describe the various thermodynamic phases of hard spherotetrahedra.

Finding the essential features $\left(\bar{q}_{l}, s\right)$ that distinguish different phases is an example of a dimensionality reduction problem. The generic problem is to project high-dimensional data onto a space of much smaller dimensions while preserving a notion of similarity: "close" structures in the high-dimensional space should remain close even in the subspace, and likewise for those "far away". A well-known technique to approach such a problem is a principal component analysis (PCA), which defines a linear basis transformation in such a way that the projection of the data onto a hopefully small subset of these socalled "principal components" carry most of the variance or information on the data. Specifically, PCA provides two directly interpretable objects: a set of eigenvalues from which the effective dimensionality of the data set can be inferred and a set of eigenvectors (particular linear combinations of $\bar{q}_{l}$ 's) that provides information on which features $\left(\bar{q}_{l}\right.$; s) are most relevant. Although PCA is a linear technique and perhaps less powerful than alternative nonlinear techniques, the advantage of PCA is that the eigenvectors, which can straightforwardly be employed as reliable order parameters, are easy to interpret.

We construct our data from the $N p T$ Monte Carlo simulations discussed above, with typically 250-256 particles in the simulation box for crystalline structures and 1968 particles for the quasicrystal approximant (QA) structure. For 25 equidistantly distributed particles shape parameters $s \in$ $\{0.02,0.04, \ldots, 0.5\}$ and for five additional ones $s \in\{0.6,0.7$, $0.8,0.9\}$, we calculate for every particle in the system the set of 11 bond order parameters $|Q\rangle \equiv\left(\bar{q}_{2}, \bar{q}_{3}, \ldots, \bar{q}_{12}\right)$, for many configurations at pressures $p$ in the interval $5 \leq p v_{\mathrm{p}} / k_{\mathrm{B}} T \leq 250$ with $v_{\mathrm{p}}$ the particle volume and $T$ the temperature. The use of the "ket-vector" notation for the one-particle state $|Q\rangle$ will become clear below. In the crystalline states for $s \geq 0.1$ we consider 55 pressures $p$ yielding packing fractions $\phi \in[0.38$, $0.88]$, and in the QA states for $0.02 \leq s \leq 0.1$ we consider 58 pressures yielding $\phi \in[0.28,0.86]$. We thus consider configurations of all phases in the phase diagram of spherotetrahedra, i.e., the fluid phase, the QA phases, and all crystal phases at close-packing and intermediate densities. This provides us with an $11 \times \mathcal{N}$ matrix of input data, where $\mathcal{N}=\sum_{\{s\}} N_{s} N_{c, s}=987180$ with $s$ the label of the considered shapes and $N_{s}$ and $N_{c, s}$ the number of particles and the total number of configurations considered for particle shape $s$, respectively. This large matrix thus contains all the information on the local structure of all particles in the various thermodynamic phases and of the various shapes considered. In fact we also considered an even higher-dimensional set of bond order parameters for every particle in the system, consisting of $\bar{q}_{l}$ supplemented by the higher-order invariant $w_{l}$ 
both with $2 \leq l \leq 24$; see SI for details. We found this larger set to yield little additional information, however, and so we keep our focus on $\bar{q}_{l}$ with $2 \leq l \leq 12$ here.

We perform a PCA on the $11 \times \mathcal{N}$ data matrix, which gives rise to a symmetric $11 \times 11$ covariance matrix, scaled such that its trace (and hence the sum of its 11 eigenvalues) equals unity. We denote the eigenvalues of the covariant matrix by $\lambda_{i}$ and its 11-dimensional eigenvectors in "bra-ket" notation by $\left|V_{i}\right\rangle$ for $i$ $=1, \ldots, 11$. Note that all eigenvalues are real since the covariant matrix is Hermitian by construction. The three largest eigenvalues are $\lambda_{1}=0.66, \lambda_{2}=0.25$, and $\lambda_{3}=0.04$, such that (loosely speaking) the three-dimensional linear subspace $\left|V_{1}\right\rangle$ $\oplus\left|V_{2}\right\rangle \oplus\left|V_{3}\right\rangle$ accounts for a fraction as large as $\lambda_{1}+\lambda_{2}+\lambda_{3}=$ 0.95 of the variance of the data, i.e., $95 \%$, which signifies a substantial dimensionality reduction. Recalling that we denoted the one-particle state by the 11-dimensional ketvector $|Q\rangle=\left(\bar{q}_{2}, \bar{q}_{3}, \ldots, \bar{q}_{12}\right)$, we can define the inner product $y_{i}$ $\equiv\left\langle V_{i} \mid Q\right\rangle$ as the projection of $|Q\rangle$ on the principal components $\left|V_{i}\right\rangle$ for $i=1,2$, 3. Instead of the projection $y_{i}$ of a one-particle state, it will also turn out to be convenient to consider its average $\left\langle y_{i}\right\rangle$ over all particles in the system (for a given shape $s$ at a given state point).

In Figure 6 we show the resulting average projections $\left\langle y_{1}\right\rangle$ (a), $\left\langle y_{2}\right\rangle(\mathrm{b})$, and $\left\langle y_{3}\right\rangle$ (c) as a function of the packing fraction

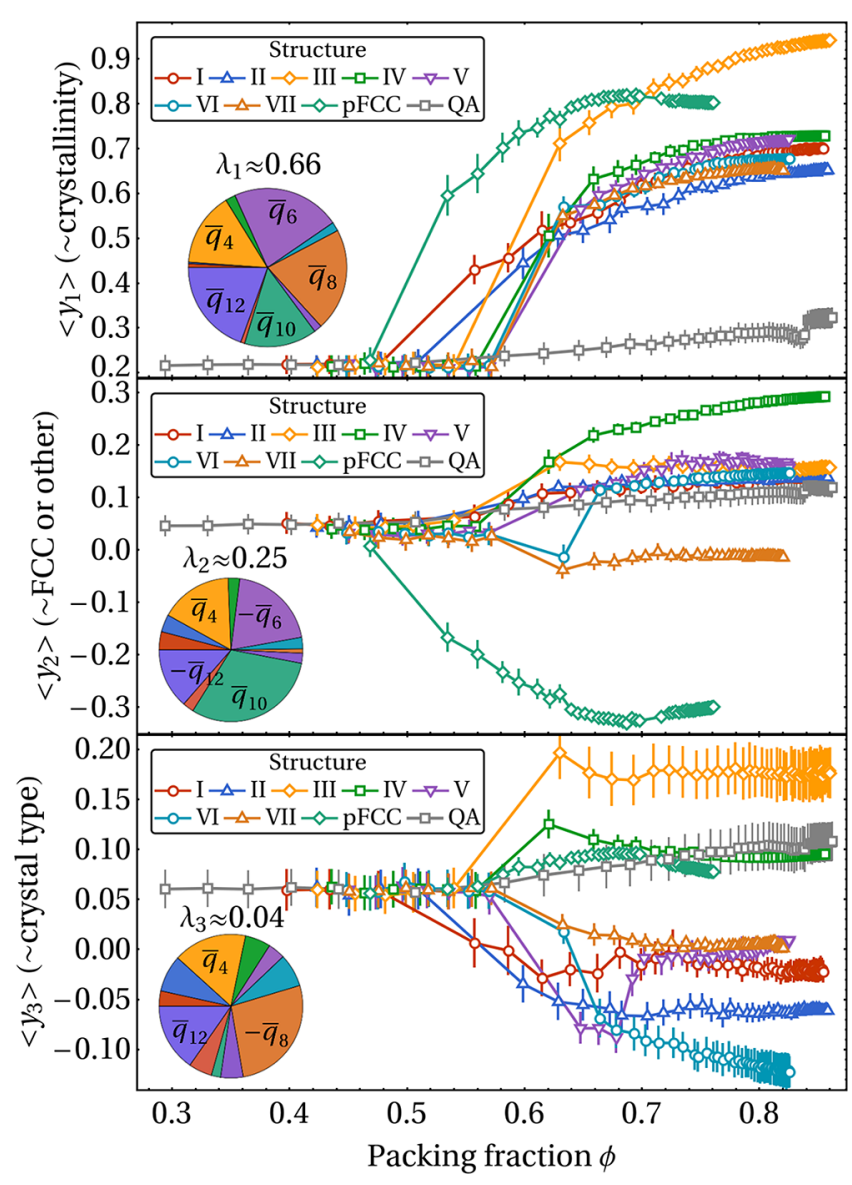

Figure 6. Global order parameters $\left\langle y_{i}\right\rangle$ with their corresponding eigenvectors (pie charts) and eigenvalues $\lambda_{i}$ obtained from PCA as a function of the packing fraction $\phi$, for various particle shapes $s$. Here $\left\langle y_{1}\right\rangle$ describes the increase of nonspecific crystalline order with increasing density, $\left\langle y_{2}\right\rangle$ distinguishes sphere-like from tetrahedron-like crystals, and $\left\langle y_{3}\right\rangle$ further distinguishes the various tetragonal crystals. $\phi$, in all three cases for eight judiciously chosen particle shapes $s \in\{0.04,0.12,0.14,0.22,0.34,0.36,0.4,0.8\}$ that exhibit at sufficiently high $\phi$ seven of the 13 close-packed crystal structures or the plastic crystal pFCC. For the quasicrystal approximant, we show data for $s=0.06$. The pie charts in Figure 6 represent the three dominant eigenvectors $\left|V_{1}\right\rangle$ (a), I $\left.V_{2}\right\rangle$ (b), and $\left|V_{3}\right\rangle$ (c), where the parts of the pie denote the weights of the 11 components $\bar{q}_{l}$ (including the relative sign).

The pie chart of the first eigenvector $\left|V_{1}\right\rangle$ with eigenvalue $\lambda_{1}$ $=0.66$ is seen to be comprised of all $\bar{q}_{l}$ with $l$ even in roughly equal measure, except for $\bar{q}_{2}$, which is virtually absent in $\left|V_{1}\right\rangle$. In other words, up to an overall scaling factor we have $y_{1} \simeq \bar{q}_{4}$ $+\bar{q}_{6}+\bar{q}_{8}+\bar{q}_{10}+\bar{q}_{12}$, where we set the five $O(1)$ coefficients exactly equal to unity for illustration purposes. The projection $y_{1}$ of the state of a particle (or its average $\left\langle y_{1}\right\rangle$ over all particles) can thus be interpreted as an overall but nonspecific measure for the degree of crystallinity of the particular state. This is confirmed by the $\phi$-dependence of $\left\langle y_{1}\right\rangle$ in Figure 6(a), which for all particle shapes is a universal (nonzero) constant in the low- $\phi$ fluid state, while any departure from this constant indicates a crystal structure. In the crystal regime, $y_{1}$ captures the increase in structural order with increasing density. Interestingly, we conclude that density is actually a good order parameter for these systems, not only because of the monotonic dependence of $\left\langle y_{1}\right\rangle$ on $\phi$ in Figure 6(a), but also because $y_{1}$ exhibits the largest variance in the data.

The pie chart of the second eigenvector $\left|V_{2}\right\rangle$ in Figure 6(b) is dominated by large positive $\bar{q}_{10}$ and $\bar{q}_{4}$ components and negative $\bar{q}_{6}$ and $\bar{q}_{12}$ components. The corresponding projection is then, again up to an overall scaling factor and with rough estimates for the coefficients, given by $y_{2} \simeq \bar{q}_{4}-\bar{q}_{6}+2 \bar{q}_{10}-$ $\bar{q}_{12}$. The eigenvector $\left|V_{2}\right\rangle$, with eigenvalue $\lambda_{2}=0.25$ and hence capturing $25 \%$ of the variance of the data, therefore mainly distinguishes 4-fold tetrahedron-like crystals (with large $\bar{q}_{4}$ and $\bar{q}_{10}$ ) from the hexagonal sphere-like ones (with large $\bar{q}_{6}$ and $\left.\bar{q}_{12}\right)$. Also this interpretation is confirmed by the density dependence of the average projection $\left\langle y_{2}\right\rangle$ on $\left|V_{2}\right\rangle$ for all nine shapes, which clearly shows a large positive result for crystal IV and many of the other tetrahedron-like crystals and (deeply) negative results for the structures pFCC and VII. Clearly, however, $\left\langle y_{2}\right\rangle$ cannot disentangle many of the 4-fold structures.

The disentanglement of many of the tetrahedron-like crystals is accomplished by the projection $\left\langle y_{3}\right\rangle$ of the data on the third eigenvector $\left|V_{3}\right\rangle$ as shown in Figure 6(c). The pie chart shows that $\left|V_{3}\right\rangle$ is dominated by a positive $\bar{q}_{8}$ component combined with negative $\bar{q}_{4}$ and $\bar{q}_{12}$ components, such that with rough estimates of the prefactors we have up to an overall constant that $y_{3} \simeq-\bar{q}_{4}+2 \bar{q}_{8}-\bar{q}_{12}$. In other words, 8-fold symmetries play a role that are not simply higher harmonics of a 4 -fold symmetry as their weights in $y_{3}$ have opposite signs compared to the weights in $y_{1}$. Even though the corresponding eigenvalue is as small as $\lambda_{3}=0.04$ such that the projection $y_{3}$ only captures $4 \%$ of the variance, the density dependence of $\left\langle y_{3}\right\rangle$ shown in Figure 6(c) separates essentially all different crystals relatively well. The resolving power of the projections $\left\langle y_{2}\right\rangle$ and $\left\langle y_{3}\right\rangle$ is best exemplified by following the lines of structures V and VI in Figure 6, both of which display jumps that respectively correspond to the solid-solid transitions $\mathrm{V} \rightarrow$ VI and VI $\rightarrow$ VII that these structures undergo before melting into the fluid, as described earlier in this study.

To summarize, we find that $y_{1} \simeq \bar{q}_{4}+\bar{q}_{6}+\bar{q}_{8}+\bar{q}_{10}+\bar{q}_{12}$, which strongly correlates with density and separates well the fluid phase and QA from the crystal structures, whereas specific 
differences in $\bar{q}_{l}$ 's effectively distinguish the different crystal structures, e.g., $y_{2} \simeq \bar{q}_{4}-\bar{q}_{6}+2 \bar{q}_{10}-\bar{q}_{12}$ separates well the tetrahedron-like crystals from the sphere-like crystals, and $y_{3} \simeq$ $-\bar{q}_{4}+2 \bar{q}_{8}-\bar{q}_{12}$ distinguishes the various tetrahedron-like crystals.

To illustrate this further, we study the distributions of the local bond order parameters, rather than the all-particleaveraged global bond order parameters. Figure 7 shows three

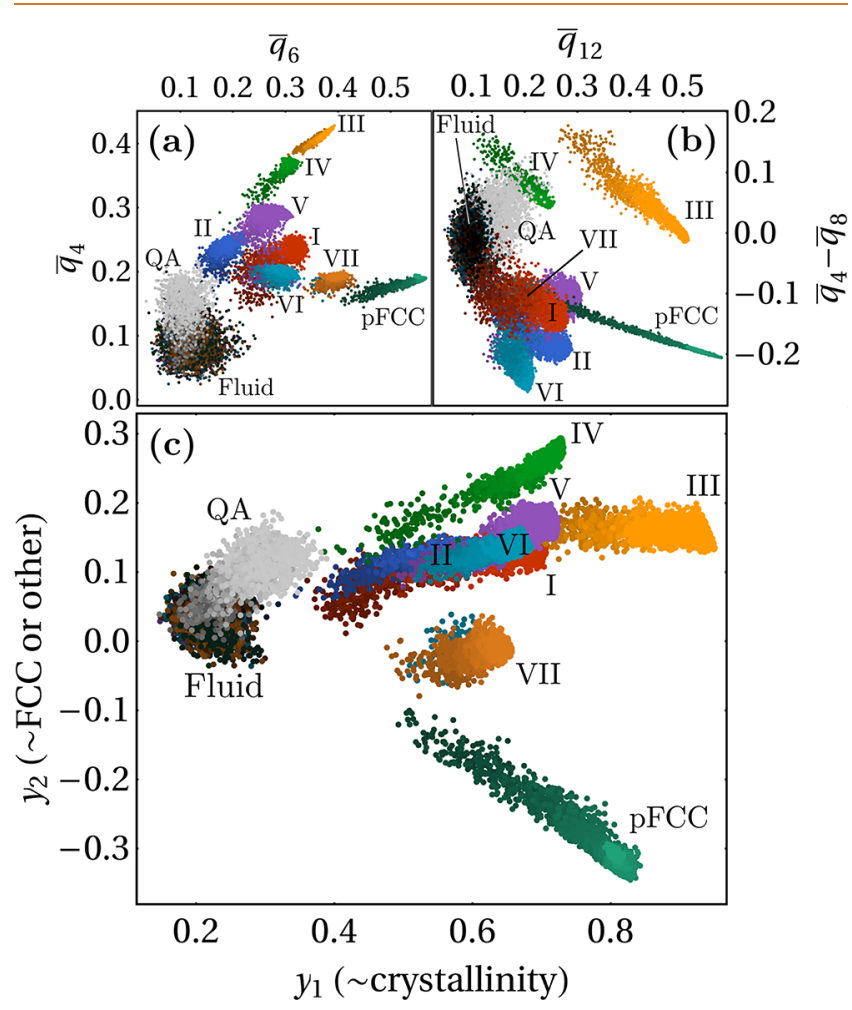

Figure 7. Distributions of local bond order parameters along (a) $\bar{q}_{4}$ and $\bar{q}_{6}$, (b) $\bar{q}_{4}-\bar{q}_{8}$ and $\bar{q}_{12}$, and (c) the first two principal axes obtained by PCA. Different structures are color-coded, while the color brightness indicates the density.

two-dimensional projections of the 11-dimensional $|Q\rangle$ space of all our data, where the different crystals are color-coded with a brightness that increases with density. In Figure 7 (a) and (b) the projections on the subspaces $\left(\bar{q}_{4}, \bar{q}_{6}\right)$ and $\left(\bar{q}_{4}-\bar{q}_{8}, \bar{q}_{12}\right)$ are shown, respectively. In line with our PCA finding that specific crystal information is mostly contained in the subspace $\left|V_{2}\right\rangle$ composed primarily of $\bar{q}_{4}, \bar{q}_{6}$, and $\bar{q}_{10}$ and in the subspace $\left|V_{3}\right\rangle$ composed of $\bar{q}_{4}, \bar{q}_{8}$, and $\bar{q}_{12}$, Figure 7 confirms that these five order parameters form pretty good combinations. The "standard" combination $\left(\bar{q}_{4}, \bar{q}_{6}\right)$ resolves nearly all relevant crystal structures, except for the significantly overlapping distributions of crystals I and VI and those of the fluid and the approximant. Taking the combination $\left(\bar{q}_{4}-\bar{q}_{8}, \bar{q}_{12}\right)$ in (b) as suggested by $\left|V_{3}\right\rangle$ resolves the overlap of the former, while the combinations $\left(\bar{q}_{4}, \bar{q}_{10}\right)$ and $\left(\bar{q}_{4}-\bar{q}_{6}, \bar{q}_{10}\right)$ as suggested by $\left|V_{2}\right\rangle$ partially resolve the latter (Figure $S 14(b)$ ). The projection of the data on the two dominant principal axes $\left|V_{1}\right\rangle$ and $\left|V_{2}\right\rangle$ is shown in Figure $7(\mathrm{c})$, where the fluid and QA are well separated from all crystal structures by the line $y_{1} \simeq 0.4$. Moreover, the sphere-like crystals (VII and pFCC) at $y_{2}<0$ are well separated from the tetrahedron-like crystals (I-VI) at $y_{2}>0$ with III and IV resolved from the other four crystal structures. In order to resolve the latter four, one could project the data on the third principal component $\left|V_{3}\right\rangle$ in line with Figure 6(c), where they are represented by the lowest four curves. Alternatively, they are well-resolved in the subspace $\left(\bar{q}_{4}\right.$, $\left.\bar{q}_{10}\right)$ as shown in the Supporting Information. Figure 7 also shows that the QA is in between the fluid and the crystal phases with a unique $\bar{q}_{l}$ fingerprint, which could for instance be used as an order parameter in umbrella sampling techniques to aid nucleation of a quasicrystal. ${ }^{57,58}$

We conclude that the (reweighted) bond order parameters $\bar{q}_{l}$ provide an excellent means of distinguishing local structures, even for the rather complex hard-particle systems studied here. Our PCA on the basis of $\bar{q}_{l}$ for $2 \leq l \leq 12$ shows not only that the expected $\bar{q}_{4}$ and $\bar{q}_{6}$ play a key role in the characterization of the self-assembled structures but that a surprisingly large role is also played by $\bar{q}_{10}$, mainly to distinguish hexagonal from tetragonal structures and the QA from the fluid and the crystal phases. Moreover, $\bar{q}_{8}$ is found to be crucial for disentangling several of the tetragonal phases, even though the corresponding eigenvalue in the PCA ( $\lambda_{3}$ in this case) is an order of magnitude smaller than the largest two eigenvalues. This example shows that the relative magnitude of an eigenvalue is not necessarily a measure for the resolving power of clusters of data points in a dimensionality reduction technique such as PCA.

\section{CONCLUSIONS AND OUTLOOK}

In summary, we have studied the phase behavior of hard spherotetrahedra, a family of shapes that interpolate between hard tetrahedra and hard spheres. Our results demonstrate the structural complexity that arises in the solid phases of these particles due to a competition between directional entropic forces from flat facets, geometric constraints to achieve the densest packing, and rotational entropy. In the close-packed limit, rounding tetrahedra enforces the formation of complex distorted lattices where symmetries are broken in order to achieve optimal packing. We have found 13 close-packed structures, most of which are significantly denser than previously reported. ${ }^{47}$ Most strikingly, we find an $N=82$ quasicrystal approximant to be the densest packing for a shape parameter $s \in[0.04,0.088]$. To the best of our knowledge, this is the largest unit cell for the densest packing of any singlecomponent hard-particle system found thus far. ${ }^{12,15,20,22,26,32,52,53}$ That the densest packing for this system is a quasicrystal approximant again raises the fascinating possibility of an aperiodic densest packing. We could not determine whether it is the periodic approximant that is densest or the aperiodic quasicrystal in this work. A more rigorous study is needed to resolve this question.

At intermediate densities, rounding the edges and vertices of tetrahedra seems to have a dramatic effect on the crystallization rate and melting density of crystal phases, to the point where we did not observe any crystallization from the fluid phase for $s$ $\in(0.16,0.5)$ even after $10^{8}$ Monte Carlo cycles. The lack of self-assembly for similarly shaped truncated tetrahedra ${ }^{33}$ was attributed to a competition between several local structural motifs. ${ }^{34}$ Given the many dense packings we observe in this region, a similar mechanism may be at play here as well. It would be interesting to see in which way truncation and rounding of polyhedra differ in the suppression and enhancement of self-assembling (quasi)crystal structures. Perhaps even greater control could be achieved by combining them.

Finally, we classified the different crystal structures with a set of bond orientational order parameters $\bar{q}_{l}$. Performing 
dimensionality reduction by means of a principal component analysis, we found that linear combinations of $\bar{q}_{l}$, s can form excellent order parameters to both detect and distinguish between the many crystal phases we observed. The sum of all even $\bar{q}_{l}$ 's with $4 \leq l \leq 12$ provides a general measure of overall crystallinity, which may prove useful in nucleation studies, enhanced sampling techniques or inverse-design methods. The "standard" combination $\left(\bar{q}_{4}, \bar{q}_{6}\right)$ works well for this system as well, resolving most crystals except for crystal structures I and VI, which overlap in this subspace. Taking the difference $\bar{q}_{4}-$ $\bar{q}_{8}$, as suggested by the third eigenvector obtained from PCA, allows one to distinguish between these two structures as well. In general, we conclude that differences between $\bar{q}_{l}$ can serve to separate structures that are otherwise poorly resolved when only considering individual bond order parameters.

\section{METHODS}

Predicting the Densest Packings. We define the spherotetrahedron shape in terms of Minkowski sums: it is the sum of a tetrahedron of edge length $L$ with a sphere of radius $R$, both centered around the origin. This is equivalent to the volume within a distance $R$ from a tetrahedron of edge length $L$. The Minkowski sum definition lends itself well to various collision/overlap detection algorithms currently in use. In our own implementation, we use the GilbertJohnson-Keerthi algorithm. ${ }^{59}$ Lacking a simple analytical expression for the volume $v_{\mathrm{p}}$ of a spheropolyhedron, we compute it numerically with a method described in the Supporting Information (SI). To determine the densest packings of spherotetrahedra as a function of the shape parameter $s$, we use the floppy-box Monte Carlo algorithm described in ref 24. In short, this algorithm finds densest packings of hard particles by numerically compressing unit cells of variable shapes with periodic boundary conditions and a small number of particles to increasingly high pressures. The reported densest packings for each particle shape (roundness $s$ ) are the result of at least 20 independent compression runs, each of which compressed either an $N=1-, 2-, 3-$, or 4-particle unit cell from a dimensionless pressure $p_{\text {start }}^{*}=\beta p V_{\mathrm{p}}=1$ to $p_{\text {end }}^{*}=\beta p V_{\mathrm{p}}=10^{7}$, with $p$ the pressure, $V_{\mathrm{p}}=N v_{\mathrm{p}}$ the volume occupied by the $N$ particles in the unit cell, and $\beta=1 / k_{\mathrm{B}} T$ with $k_{\mathrm{B}}$ Boltzmann's constant and $T$ the temperature. To sample different compression routes, we increased the pressure of each MC cycle $m$ as $p_{m}^{*}=p_{\text {start }}^{*}+\left(p_{\text {end }}^{*}-p_{\text {start }}^{*}\right)(m / M)^{\gamma}$ over a total of $M=10^{7}$ MC cycles. The exponent $\gamma$ was set randomly within the interval $5.2 \leq \gamma \leq 9$. These values seemed to provide the best sampling to reliably reproduce the known dimer crystal packing of tetrahedra, and we made the assumption that this provides good sampling for other shapes as well. The space group of the crystal was identified using FindSym ${ }^{60,61}$ on the obtained primitive unit cell.

Bond-Orientational Order Parameters. To describe the local environment of each particle, we employ a reweighted variation of the Steinhardt bond-orientational order parameters ${ }^{49}$ known as Minkowski structure metrics, ${ }^{51}$ where the bonds between particles are defined through a Voronoi construction and the bond order parameters $q_{l m}$ are reweighted by the area of the shared Voronoi facet:

$$
\begin{aligned}
& q_{l m}(i)=\sum_{j} \frac{a_{i j}}{A_{i}} Y_{l m}\left(\boldsymbol{r}_{i j}\right) \\
& q_{l}(i)=\sqrt{\frac{4 \pi}{2 l+1} \sum_{m=-l}^{l}\left|q_{l m}(i)\right|^{2}}
\end{aligned}
$$

Here, $Y_{l m}$ are the spherical harmonics, $a_{i j}$ is the area of the Voronoi facet shared by particles $i$ and $j, r_{i j}$ is the bond vector from $i$ to $j$, and $A_{i}$ is the total area of the Voronoi cell of $i$. Defining bonds through the Voronoi construction makes the method parameter-free, and weighting by the shared facet area makes the metrics less susceptible to fluctuations in the number of bonds. Analogous to the averaged Steinhardt bond order parameters defined in ref 50, we use averaged
Minkowski structure metrics by simply averaging the $q_{l m}$ (eq 1 ) over the neighbors before calculating the rotationally invariant $\bar{q}_{l}$ :

$$
\bar{q}_{l m}(i)=\frac{1}{\tilde{N}_{\mathrm{b}}(i)} \sum_{k=0}^{\widetilde{N}_{b}(i)} q_{l m}(k)
$$

where $\tilde{N}_{\mathrm{b}}(i)$ denotes the set of neighbors of $i$, and $i$ itself, and $q_{l m}$ denotes the nonaveraged structure metrics. The averaged rotationally invariant $\bar{q}_{l}$ are then obtained by simply replacing $q_{l m}$ with $\overline{\bar{q}}_{l m}$ :

$$
\bar{q}_{l}(i)=\sqrt{\frac{4 \pi}{2 l+1} \sum_{m=-l}^{l}\left|\bar{q}_{l m}(i)\right|^{2}}
$$

In our analysis we considered $l \leq 24$ except $\bar{q}_{0}$ and $\bar{q}_{1}$, which are respectively always 1 and $0 .{ }^{51}$ Our implementation for the calculation of these structure metrics is available on Github. ${ }^{62}$

Self-Assembly and Equation of State Simulations. To probe the self-assembly behavior and to calculate the equations of state, we performed simulations using HOOMD-blue's ${ }^{63,64}$ hard-particle Monte Carlo (HPMC) ${ }^{65}$ module. We used MC simulations in the NVT ensemble using $10^{8} \mathrm{MC}$ cycles to study the self-assembly. A relatively large number of MC cycles is needed, as previous studies showed that the formation of the quasicrystal of hard tetrahedra is quite slow. ${ }^{21,23,54}$ Particles were allowed to translate and rotate with a variable step size such that the acceptance rate of such moves was $30 \%$. To calculate the equations of state, we performed MC simulations of $N \approx 400$ particles in a constant-pressure ensemble, where in addition to particle moves and rotations the simulation box was allowed to change its volume and to shear. The step sizes of these latter moves were tuned to an acceptance ratio of $15 \%$. We used $10^{7}$ $\mathrm{MC}$ cycles for equilibration and $10^{5} \mathrm{MC}$ cycles for sampling. Initial configurations were either a low-density fluid, a high-density crystal whose unit cell we obtained from the FBMC simulations, or the quasicrystal approximant.

\section{ASSOCIATED CONTENT}

\section{Supporting Information}

The Supporting Information is available free of charge at https://pubs.acs.org/doi/10.1021/acsnano.0c05288.

Additional details from the crystal structure analysis, evidence for the plastic and quasicrystal phases, an expanded analysis based on $\bar{q}_{l}$ supplemented by $w_{l}$ with $l$ $\leq 24$ (PDF)

Unit cells of all densest packing (ZIP)

Python script to calculate the volume of spheropolyhedra (TXT)

\section{AUTHOR INFORMATION}

\section{Corresponding Authors}

Robin van Damme - Soft Condensed Matter, Debye Institute for Nanomaterials Science, Utrecht University, 3584 CC Utrecht, The Netherlands; $\odot$ orcid.org/0000-0003-28713726; Email: r.vandamme@uu.nl

Marjolein Dijkstra - Soft Condensed Matter, Debye Institute for Nanomaterials Science, Utrecht University, 3584 CC Utrecht, The Netherlands; Email: m.dijkstra@uu.nl

\section{Authors}

Gabriele M. Coli - Soft Condensed Matter, Debye Institute for Nanomaterials Science, Utrecht University, 3584 CC Utrecht, The Netherlands; 10 orcid.org/0000-0002-5125-8007

René van Roij - Institute for Theoretical Physics, Utrecht University, 3584 CC Utrecht, The Netherlands

Complete contact information is available at: https://pubs.acs.org/10.1021/acsnano.0c05288 


\section{Notes}

The authors declare no competing financial interest.

\section{ACKNOWLEDGMENTS}

This work is part of the D-ITP consortium, a program of The Netherlands Organisation for Scientific Research (NWO) that is funded by the Dutch Ministry of Education, Culture and Science (OCW). We acknowledge financial support from an NWO-VICI grant.

\section{REFERENCES}

(1) Glotzer, S. C.; Solomon, M. J. Anisotropy of Building Blocks and Their Assembly Into Complex Structures. Nat. Mater. 2007, 6, 557562.

(2) Zhuang, Z.; Peng, Q.; Wang, X.; Li, Y. Tetrahedral Colloidal Crystals of Ag2S Nanocrystals. Angew. Chem., Int. Ed. 2007, 46, 8174-8177.

(3) Sacanna, S.; Pine, D. J. Shape-Anisotropic Colloids: Building Blocks for Complex Assemblies. Curr. Opin. Colloid Interface Sci. 2011, $16,96-105$

(4) Miszta, K.; de Graaf, J.; Bertoni, G.; Dorfs, D.; Brescia, R.; Marras, S.; Ceseracciu, L.; Cingolani, R.; van Roij, R.; Dijkstra, M.; Manna, L. Hierarchical self-assembly of suspended branched colloidal nanocrystals into superlattice structures. Nat. Mater. 2011, 10, 872876.

(5) Henzie, J.; Grünwald, M.; Widmer-Cooper, A.; Geissler, P. L.; Yang, P. Self-Assembly of Uniform Polyhedral Silver Nanocrystals Into Densest Packings and Exotic Superlattices. Nat. Mater. 2012, 11, 131-137.

(6) Huang, M. H.; Lin, P.-H. Shape-Controlled Synthesis of Polyhedral Nanocrystals and Their Facet-Dependent Properties. Adv. Funct. Mater. 2012, 22, 14-24.

(7) Boles, M. A.; Engel, M.; Talapin, D. V. Self-Assembly of Colloidal Nanocrystals: From Intricate Structures to Functional Materials. Chem. Rev. 2016, 116, 11220-11289.

(8) Two-Dimensionally Patterned Nanostructures Based on Monolayer Colloidal Crystals: Controllable Fabrication, Assembly, and Applications. Nano Today 2011, 6, 608-631.

(9) Kim, S.-H.; Lee, S. Y.; Yang, S.-M.; Yi, G.-R. Self-Assembled Colloidal Structures for Photonics. NPG Asia Mater. 2011, 3, 25-33.

(10) Choi, J.-H.; Wang, H.; Oh, S. J.; Paik, T.; Sung, P.; Sung, J.; Ye, X.; Zhao, T.; Diroll, B. T.; Murray, C. B.; Kagan, C. R. Exploiting the Colloidal Nanocrystal Library to Construct Electronic Devices. Science 2016, 352, 205-208.

(11) Stein, A.; Wilson, B. E.; Rudisill, S. G. Design and Functionality of Colloidal-Crystal-Templated Materials - Chemical Applications of Inverse Opals. Chem. Soc. Rev. 2013, 42, 2763-2803.

(12) Torquato, S.; Jiao, Y. Dense Packings of the Platonic and Archimedean Solids. Nature 2009, 460, 876-879.

(13) Agarwal, U.; Escobedo, F. A. Mesophase Behaviour of Polyhedral Particles. Nat. Mater. 2011, 10, 230-235.

(14) Smallenburg, F.; Filion, L.; Marechal, M.; Dijkstra, M. VacancyStabilized Crystalline Order in Hard Cubes. Proc. Natl. Acad. Sci. U. S. A. 2012, 109, 17886-17890.

(15) Gantapara, A. P.; de Graaf, J.; van Roij, R.; Dijkstra, M. Phase Diagram and Structural Diversity of a Family of Truncated Cubes: Degenerate Close-Packed Structures and Vacancy-Rich States. Phys. Rev. Lett. 2013, 111, No. 015501.

(16) Gantapara, A. P.; de Graaf, J.; van Roij, R.; Dijkstra, M. Phase Behavior of a Family of Truncated Hard Cubes. J. Chem. Phys. 2015, 142, No. 054904.

(17) van der Meer, B.; van Damme, R.; Dijkstra, M.; Smallenburg, F.; Filion, L. Revealing a Vacancy Analog of the Crowdion Interstitial in Simple Cubic Crystals. Phys. Rev. Lett. 2018, 121, 258001.

(18) Kepler, J. Strena Seu de Niue Sexangula; Gottfried Tampach, 1966.

(19) Jaeger, H. M.; Nagel, S. R.; Behringer, R. P. Granular Solids, Liquids, and Gases. Rev. Mod. Phys. 1996, 68, 1259-1273.
(20) Torquato, S.; Jiao, Y. Dense Packings of Polyhedra: Platonic and Archimedean Solids. Phys. Rev. E 2009, 80, No. 041104.

(21) Haji-Akbari, A.; Engel, M.; Keys, A. S.; Zheng, X.; Petschek, R. G.; Palffy-Muhoray, P.; Glotzer, S. C. Disordered, Quasicrystalline and Crystalline Phases of Densely Packed Tetrahedra. Nature 2009, 462, 773-777.

(22) de Graaf, J.; van Roij, R.; Dijkstra, M. Dense Regular Packings of Irregular Nonconvex Particles. Phys. Rev. Lett. 2011, 107, 155501.

(23) Haji-Akbari, A.; Engel, M.; Glotzer, S. C. Phase Diagram of Hard Tetrahedra. J. Chem. Phys. 2011, 135, 194101.

(24) de Graaf, J.; Filion, L.; Marechal, M.; van Roij, R.; Dijkstra, M. Crystal-Structure Prediction via the Floppy-Box Monte Carlo Algorithm: Method and Application to Hard (Non)convex Particles. J. Chem. Phys. 2012, 137, 214101.

(25) Batten, R. D.; Stillinger, F. H.; Torquato, S. Phase Behavior of Colloidal Superballs: Shape Interpolation from Spheres to Cubes. Phys. Rev. E 2010, 81, No. 061105.

(26) Ni, R.; Gantapara, A. P.; de Graaf, J.; van Roij, R.; Dijkstra, M. Phase Diagram of Colloidal Hard Superballs: From Cubes via Spheres to Octahedra. Soft Matter 2012, 8, 8826.

(27) Marechal, M.; Zimmermann, U.; Löwen, H. Freezing of Parallel Hard Cubes With Rounded Edges. J. Chem. Phys. 2012, 136, 144506.

(28) Rossi, L.; Soni, V.; Ashton, D. J.; Pine, D. J.; Philipse, A. P.; Chaikin, P. M.; Dijkstra, M.; Sacanna, S.; Irvine, W. T. M. ShapeSensitive Crystallization in Colloidal Superball Fluids. Proc. Natl. Acad. Sci. U. S. A. 2015, 112, 5286-5290.

(29) Wang, D.; Hermes, M.; Kotni, R.; Wu, Y.; Tasios, N.; Liu, Y.; De Nijs, B.; Van Der Wee, E. B.; Murray, C. B.; Dijkstra, M.; van Blaaderen, A. Interplay Between Spherical Confinement and Particle Shape on the Self-Assembly of Rounded Cubes. Nat. Commun. 2018, 9.

(30) Khadilkar, M. R.; Agarwal, U.; Escobedo, F. A. Phase Behavior of Binary Mixtures of Hard Convex Polyhedra. Soft Matter 2013, 9, 11557.

(31) Damasceno, P. F.; Engel, M.; Glotzer, S. C. Crystalline Assemblies and Densest Packings of a Family of Truncated Tetrahedra and the Role of Directional Entropic Forces. ACS Nano 2012, 6, 609-614.

(32) Chen, E. R.; Klotsa, D.; Engel, M.; Damasceno, P. F.; Glotzer, S. C. Complexity in Surfaces of Densest Packings for Families of Polyhedra. Phys. Rev. X 2014, 4, No. 011024.

(33) Klotsa, D.; Chen, E. R.; Engel, M.; Glotzer, S. C. Intermediate Crystalline Structures of Colloids in Shape Space. Soft Matter 2018, 14, 8692-8697.

(34) Teich, E. G.; van Anders, G.; Glotzer, S. C. Identity Crisis in Alchemical Space Drives the Entropic Colloidal Glass Transition. Nat. Commun. 2019, 10, 64.

(35) Greyson, E. C.; Barton, J. E.; Odom, T. W. Tetrahedral Zinc Blende Tin Sulfide Nano- and Microcrystals. Small 2006, 2, 368-371.

(36) Nagaoka, Y.; Zhu, H.; Eggert, D.; Chen, O. Single-Component Quasicrystalline Nanocrystal Superlattices Through Flexible Polygon Tiling Rule. Science 2018, 362, 1396-1400.

(37) Nagaoka, Y.; Tan, R.; Li, R.; Zhu, H.; Eggert, D.; Wu, Y. A.; Liu, Y.; Wang, Z.; Chen, O. Superstructures Generated From Truncated Tetrahedral Quantum Dots. Nature 2018, 561, 378-382.

(38) Stöber, W.; Fink, A.; Bohn, E. Controlled Growth of Monodisperse Silica Spheres in the Micron Size Range. J. Colloid Interface Sci. 1968, 26, 62-69.

(39) Graf, C.; Vossen, D. L.; Imhof, A.; van Blaaderen, A. A General Method to Coat Colloidal Particles With Silica. Langmuir 2003, 19, $6693-6700$

(40) Hales, T.; Adams, M.; Bauer, G.; Dang, T. D.; Harrison, J.; Le Truong, H.; Kaliszyk, C.; Magron, V.; McLaughlin, S.; Nguyen, T. T.; Nguyen, Q. T.; Nipkow, T.; Obua, S.; Pleso, J.; Rute, J.; Solovyev, A.; Ta, T. H. A.; Tran, N. T.; Trieau, T. D.; Urban, J.; et al. A Formal Proof of the Kepler Conjecture. Forum Math. Pi 2017, 5, No. e2.

(41) Conway, J. H.; Torquato, S. Packing, Tiling, and Covering With Tetrahedra. Proc. Natl. Acad. Sci. U. S. A. 2006, 103, 10612-10617. 
(42) Chen, E. R. A Dense Packing of Regular Tetrahedra. Discrete Comput. Geom. 2008, 40, 214-240.

(43) Kallus, Y.; Elser, V.; Gravel, S. Dense Periodic Packings of Tetrahedra with Small Repeating Units. Discrete Comput. Geom. 2010, $44,245-252$.

(44) Torquato, S.; Jiao, Y. Analytical Constructions of a Family of Dense Tetrahedron Packings and the Role of Symmetry. arXiv.org, ePrint Arch., Condens. Matter 2010, arXiv:0912.4210. https://arxiv.org/ abs/0912.4210 (accessed April 24, 2019).

(45) Chen, E. R.; Engel, M.; Glotzer, S. C. Dense Crystalline Dimer Packings of Regular Tetrahedra. Discrete Comput. Geom. 2010, 44, 253-280.

(46) Gravel, S.; Elser, V.; Kallus, Y. Upper Bound on the Packing Density of Regular Tetrahedra and Octahedra. Discrete Comput. Geom. 2011, 46, 799-818.

(47) Jin, W.; Lu, P.; Li, S. Evolution of the Dense Packings of Spherotetrahedral Particles: From Ideal Tetrahedra to Spheres. Sci. Rep. 2015, 5, 15640.

(48) Haji-Akbari, A.; Chen, E. R.; Engel, M.; Glotzer, S. C. Packing and Self-Assembly of Truncated Triangular Bipyramids. Phys. Rev. E 2013, 88, No. 012127.

(49) Steinhardt, P. J.; Nelson, D. R.; Ronchetti, M. BondOrientational Order in Liquids and Glasses. Phys. Rev. B: Condens. Matter Mater. Phys. 1983, 28, 784-805.

(50) Lechner, W.; Dellago, C. Accurate Determination of Crystal Structures Based on Averaged Local Bond Order Parameters. J. Chem. Phys. 2008, 129, 114707.

(51) Mickel, W.; Kapfer, S. C.; Schröder-Turk, G. E.; Mecke, K. Shortcomings of the Bond Orientational Order Parameters for the Analysis of Disordered Particulate Matter. J. Chem. Phys. 2013, 138.

(52) Jiao, Y.; Stillinger, F. H.; Torquato, S. Optimal Packings of Superballs. Phys. Rev. E 2009, 79, No. 041309.

(53) Kallus, Y.; Elser, V. Dense-Packing Crystal Structures of Physical Tetrahedra. Phys. Rev. E: Stat., Nonlinear, Soft Matter Phys. 2011, 83.

(54) Haji-Akbari, A. Thermodynamics of the Hard Tetrahedron System. Ph.D. thesis, University of Michigan, 2012.

(55) ten Wolde, P. R.; Ruiz-Montero, M. J.; Frenkel, D. Numerical Calculation of the Rate of Crystal Nucleation in a Lennard-Jones System at Moderate Undercooling. J. Chem. Phys. 1996, 104, 99329947.

(56) Kumar, R.; Coli, G. M.; Dijkstra, M.; Sastry, S. Inverse Design of Charged Colloidal Particle Interactions for Self Assembly Into Specified Crystal Structures. J. Chem. Phys. 2019, 151, No. 084109.

(57) Torrie, G.; Valleau, J. Nonphysical Sampling Distributions in Monte Carlo Free-Energy Estimation: Umbrella Sampling. J. Comput. Phys. 1977, 23, 187-199.

(58) Kästner, J. Umbrella Sampling. Wiley Interdiscip. Rev.: Comput. Mol. Sci. 2011, 1, 932-942.

(59) Gilbert, E.; Johnson, D.; Keerthi, S. A Fast Procedure for Computing the Distance Between Complex Objects in ThreeDimensional Space. IEEE Robot. Autom. Lett. 1988, 4, 193-203.

(60) Stokes, H. T.; Hatch, D. M.; Campbell, B. J. FINDSYM, version 7.0; Brigham Young University: Provo, UT, 2019. iso.byu.edu.

(61) Stokes, H. T.; Hatch, D. M. FINDSYM: Program for Identifying the Space-Group Symmetry of a Crystal. J. Appl. Crystallogr. 2005, 38, 237-238.

(62) van Damme, R. Minkowski Structure Metric Calculator, version

1.0.0; Utrecht University: Utrecht, 2019; git.io/JTtP7.

(63) Anderson, J. A.; Lorenz, C. D.; Travesset, A. General Purpose Molecular Dynamics Simulations Fully Implemented on Graphics Processing Units. J. Comput. Phys. 2008, 227, 5342-5359.

(64) Glaser, J.; Nguyen, T. D.; Anderson, J. A.; Lui, P.; Spiga, F.; Millan, J. A.; Morse, D. C.; Glotzer, S. C. Strong Scaling of GeneralPurpose Molecular Dynamics Simulations on GPUs. Comput. Phys. Commun. 2015, 192, 97-107.

(65) Anderson, J. A.; Eric Irrgang, M.; Glotzer, S. C. Scalable Metropolis Monte Carlo for Simulation of Hard Shapes. Comput. Phys. Commun. 2016, 204, 21-30. 